Journal of

Accident and

Emergency

Medicine 1994

11, 195-197

\title{
Non-penetrating corneal foreign body injuries: factors affecting delay in rehabilitation of patients
}

\author{
D.G.R.JAYAMANNE \& R.W.D.BELL
}

Departments of Ophthalmology, North Riding Infirmary, Middlesbrough, and Newcastle General Hospital, Newcastle upon Tyne

\section{SUMMARY}

A review of 783 patients with non-penetrating, superficial corneal foreign bodies (FBs), indicated that delay in rehabilitation was related to two factors: (1) the size of the abrasion following removal of the $\mathrm{FB}$, larger abrasions requiring longer diration of antibiotic ointment, and (2) inadequate removal of corneal rust. Allergy to chloramphenicol $1 \%$ ointment (5.5 in 1000), commonly used in the management of corneal abrasions, is unpredictable and can also impair rapid rehabilitation.

Key words: corneal abrasion, foreign body, rehabilitation, rust ring

\section{INTRODUCTION}

A superficial corneal FB is a common injury presenting to accident and emergency (A\&E) departments (Fig. 1). The importance of treating these injuries with adequate topical anaesthesia and instrumentation has been emphasized, ${ }^{1}$ so that the FBs can be removed with minimal trauma in an aseptic method. The majority of such injuries are industrial accidents, occuring either whilst grinding or drilling.

Rapid rehabilitation of these individuals ensures that time lost from employment is kept to a minimum. It was felt that factors which may influence the time period of healing included the size of the corneal epithelial defect (i.e. the degree of local trauma) following FB removal, and the presence of deep corneal rust. The economic implications of a prolonged recovery period and a delay in return to work in these patients, is of great importance.

\section{METHODS}

A total of 783 new patients, treated at the North Riding Infirmary, Middlesbrough with non-penetrating corneal FBs were studied prospectively, to obtain information regarding factors responsible for delay in rehabilitation. All patients initially received a careful biomicroscopic examination, and the FBs were removed, under slit lamp magnification, with a sharp green or orange needle. Topical anaesthesia was achieved using amethocaine $1 \%$, or benoxinate $0.4 \%$ drops. Subsequent treatment was governed by the biomicroscopic observations following removal of the FB.

All patients were divided into three categories (Table 1), according to the corneal features after removal of the FB. Category I consisted of patients with no corneal abrasions. Category II consisted of patients with corneal abrasions of varying sizes. Category III consisted of patients with corneal abrasions and residual deep corneal rust.

Recording of the size of the corneal abrasions was made after removal of the FB. This was carried out by comparison of maximum and minimum dimensions with the slit beam of a Haag-Streit 900 slit lamp and graded as follows: small $(\mathrm{S})<1.00$ $\mathrm{mms}$, medium (M) 1.00-1.50 mms and large $(\mathrm{L})>$ $1.50 \mathrm{mms}$. Fluorescein was used to stain the cornea.

Patients with corneal abrasions were treated with chloramphenicol $1 \%$ ointment four times daily, ${ }^{2}$ and were advised to continue treatment for up to 1 week. Eye pads were not used as separate studies have shown delayed healing with the use of pads and no improvement in patient comfort. ${ }^{3,4}$ All patients were advised to take adequate oral analgesia, such as paracetamol or cocodamol, and topical anaesthetic agents were avoided as they are known to be toxic to the corneal epithelium.

Instructions were given to patients to return to the A\&E department if symptoms worsened or had not improved after 3 days. The number of patients revisiting the department were recorded, this indicating a prolonged recovery. 
D.G.R. Jayamanne

\& R.W.D. Bell

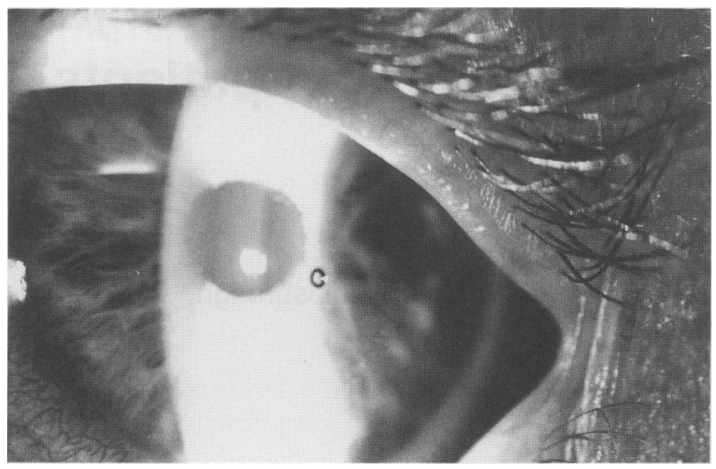

Fig. 1. Slit lamp photograph showing corneal rust ring remaining after removal of superficial ferrous foreign body, sustained whilst grinding metal.

\section{RESULTS}

The majority of patients returning to the A\&E department complained of persistent FB sensation, photophobia and lacrimation.

Table 1 shows that no patients from Category I revisited the A\&E department. $A$ total of 23 patients returned for review. Of these, four were diagnosed as having allergy to chloramphenicol (incidence 5.5 in 1000), which cleared promptly on withdrawal of medication; these patients were excluded from the statistical analysis.

Nineteen patients had persistent corneal abrasions accounting for their symptoms of FB sensation on the second visit to the department. Of these, 10 were from Category II (1.5\% of Category II patients) and nine were from Category III (15\% of category III patients). Initial corneal abrasions in all 19 patients were greater than $1.00 \mathrm{mms}$, and in 17 , they were larger than $1.5 \mathrm{mms}$ (chi square, $P<0.001$ ).

Reassurance and further treatment with chloramphenicol ointment was adequate for the category II patients with persisting corneal abrasions. The nine patients from category III all required additional removal of residual corneal rust. In this study no patients attended on a third occasion, either voluntarily or by request.

\section{DISCUSSION}

A superficial corneal FB is a very common, usually industrial, injury which can vary in severity from trivial and asymptomatic to incapacitating, with symptoms of intense FB sensation, photophobia and lacrimation.

If dealt with appropriately, there are usually no long-term sequelae and recovery is rapid, over $24-72 \mathrm{~h}$. This is confirmed by the fact that in this study, only 23 patients out of $783(2.9 \%)$ returned with persisting complaints.

It has been shown that delay in rehabilitation following non-penetrating superficial corneal FB, is related to two factors: (1) the size of the corneal epithelial defect following FB removal and (2) inadequate removal of corneal rust (chi square, $P<0.001$ ).

Corneal epithelial wounds heal by a process of cell migration and proliferation. For abrasions which involve less than $60 \%$ of the total corneal surface area, there is an exponential decrease in wound size with time and one would expect such wounds to heal within 1-4 days. ${ }^{5}$

Larger abrasions require longer duration of antibiotic ointment of up to 1 week. The use of ophthalmic ointment in preference to eye drops, permits a longer contact time between the antibiotic and the ocular surface. The ointment also acts as a lubricant between the abraded surface and the upper lid.

Topical mydriatics such as cyclopentolate $1 \%$, were not used in this study. However they are advocated if there is severe pain due to ciliary spasm. Eye pads and topical anaesthetic agents were avoided as these tend to delay epithelial healing.

Attempts should be made to remove the majority

Table 1. Results of the study

\begin{tabular}{|c|c|c|c|c|c|c|c|}
\hline \multirow[b]{2}{*}{ Category } & \multirow[b]{2}{*}{ No of patients } & \multirow{2}{*}{$\begin{array}{l}\text { Removal of } \\
\text { corneal FB }\end{array}$} & \multicolumn{3}{|c|}{$\begin{array}{c}\text { Size of abrasion } \\
\text { on fluorescein } \\
\text { staining }\end{array}$} & \multirow{2}{*}{$\begin{array}{l}\text { Chloramphenicol } \\
1 \% \text { ointment }\end{array}$} & \multirow{2}{*}{$\begin{array}{l}\text { No. of patients } \\
\text { revisiting A\&E }\end{array}$} \\
\hline & & & $\mathrm{S}$ & $M$ & $\mathrm{~L}$ & & \\
\hline 1 & 51 & Total & 0 & 0 & 0 & No & 0 \\
\hline II & 672 & Total & 633 & 22 & 19 & Yes & 12 \\
\hline III & 60 & Residual Rust & 48 & 5 & 7 & Yes & 11 \\
\hline
\end{tabular}


Non-penetrating corneal FB injuries of rust at the initial visit, ideally under slit lamp magnification. However a small amount of rust staining is an acceptable alternative to excessive corneal trauma during attempted removal, and usually causes no problems.

Care should be taken with deep centrally placed rust rings in line with the visual axis. In these cases, over-zealous debridement which breaches the subepithelial Bowman's layer can lead to permanent scarring and visual loss. Several visits may be necessary, with the liberal use of antibiotic ointment, which has the additional benefit of softening the rust, thereby facilitating removal.

\section{REFERENCES}

1. Reich J.A. (1990) Removal of corneal foreign bodies. Australian Family Physician 1 (19), 719-721.

2. Editorial. (1987) Traumatic corneal abrasion. Lancet ii, 1250.

3. Hulbert M.F.G. (1991) Efficacy of eye pad in corneal healing after corneal foreign body removal. Lancet 337 , 643.

4. Kirkpatrick J.N.P. Hoh H.B. \& Cook S.D. (1993) No eye pad for corneal abrasion. Eye 7, 468-471.

5. Dua H.S. \& Forrester J.V. (1990) The corneoscleral limbus in human corneal epithelial wound healing. American Journal of Ophthalmology 110, 646-656. 